\title{
Механизмы токопереноса в полупроводниковой структуре с пленкой пористого кремния, сформированной металл-стимулированным травлением
}

\author{
(C) Н.Н. Мельник ${ }^{1}$, В.В. Трегулов ${ }^{2}$, В.Г. Литвинов ${ }^{3}$, А.В. $^{\text {Ермачихин }}{ }^{3,}$, \\ Е.П. Трусов ${ }^{3}$, Г.Н. Скопцова ${ }^{2}$, А.И. Иванов ${ }^{2}$ \\ ${ }^{1}$ Физический институт им. П.Н. Лебедева Российской академии наук, \\ 119991 Москва, Россия \\ ${ }^{2}$ Рязанский государственный университет им. С.А. Есенина, \\ 390000 Рязань, Россия \\ ${ }^{3}$ Рязанский государственный радиотехнический университет им. В.Ф. Уткина, \\ 390005 Рязань, Россия \\ "E-mail: al.erm@mail.ru
}

Поступила в Редакцию 5 декабря 2021 г.

В окончательной редакции 10 декабря 2021 г.

Принята к публикации 25 декабря 2021 г.

\begin{abstract}
Показано, что при создании пленки пористого $\mathrm{Si}$, сформированной металл-стимулированным травлением, на монокристаллической подложке $\mathrm{Si}$ p-типа образуется барьерный слой. Выпрямляющие свойства полупроводниковой структуры можно объяснить фиксацией уровня Ферми в приповерхностном слое пористого Si вследствие высокой концентрации электрически активных дефектов (глубоких центров или ловушек), что вызывает изгиб энергетических зон и возникновение потенциального барьера. Исследование комбинационного рассеяния света показало отсутствие размерных эффектов и изменения ширины запрещенной зоны в пленке пористого $\mathrm{Si}$. В результате исследования температурной зависимости вольт-амперных характеристик и методом релаксационной спектроскопии глубоких уровней определены энергии активации глубоких центров.
\end{abstract}

Ключевые слова: пористый кремний, глубокий уровень, метод комбинационного рассеяния света, вольтамперные характеристики, релаксационная спектроскопия глубоких уровней.

DOI: 10.21883/FTP.2022.04.52197.9782

\section{1. Введение}

В настоящее время наблюдается значительный интерес в области исследований свойств пленок пористого кремния (por-Si) с целью их применения в различных областях науки и техники. Одной из наиболее важных областей применения пленок por-Si является создание антиотражающих покрытий кремниевых фотоэлектрических преобразователей солнечной энергии [1]. Для солнечной энергетики актуально использование пленок por-Si, изготовленных методом металл-стимулированного травления. Указанный метод обеспечивает наиболее низкую отражательную способность поверхности пленки por-Si по сравнению с другими методами (анодного электрохимического травления, химического окрашивающего травления), что способствует повышению эффективности солнечных элементов [1-3]. Основой метода металл-стимулированного травления является селективное химическое травление кремния с использованием предварительно осажденных на его поверхность частиц благородных металлов, наиболее часто используют серебро и золото. Травление кремния протекает непосредственно под частицами металла в растворах, содержащих плавиковую кислоту и окислитель. В результате формируется пленка por- $\mathrm{Si}$, состоящая из нитеобразных кремниевых кристаллитов $[4,5]$.
Пленки por-Si, сформированные металл-стимулированным травлением, могут применяться для создания структур, реализующих эффект усиления поверхностью комбинационного рассеяния света (SERS), повышающих чувствительность молекулярного анализа [6,7]. Кроме того, такие пленки могут использоваться для создания высокоэффективных термопреобразователей и анодов литий-ионных аккумуляторов [6,8]. Указанные области применения пленок por-Si, выращенных металл-стимулированным травлением, в настоящее время актуальны. В связи с указанным обстоятельством актуальна проблема изучения физических процессов, протекающих в полупроводниковых структурах, содержащих такие пленки. Основной задачей данной работы является разработка модели механизмов токопереноса в полупроводниковой структуре с пленкой por-Si, изготовленной методом металл-стимулированного травления, учитывающей особенности микроструктуры пористой пленки и влияние ловушек с глубокими энергетическими уровнями.

\section{2. Образцы и методы исследования}

Пленка por-Si выращивалась методом металл-стимулированного травления с использованием частиц сереб- 
ра на кремниевой монокристаллической пластине $p$-типа проводимости с удельным сопротивлением 1 Ом·см и ориентацией поверхности (100). В ходе первого этапа технологического процесса на $\mathrm{Si}$-пластине осаждались частицы серебра из раствора: $\mathrm{Ag}_{2} \mathrm{SO}_{4}(0.01 \mathrm{M}), \mathrm{HF}$ $(46 \%), \mathrm{C}_{2} \mathrm{H}_{5} \mathrm{OH}(92 \%)$ с соотношением компонентов $1: 0.1: 0.3$. Капля раствора наносилась на одну из поверхностей пластины и выдерживалась в течение $30 \mathrm{c}$. На втором этапе формировалась пористая структура. Пластина с предварительно нанесенными частицами серебра погружались в раствор: $\mathrm{H}_{2} \mathrm{O}_{2}(1.24 \mathrm{M})$, $\mathrm{HF}(46 \%), \mathrm{C}_{2} \mathrm{H}_{5} \mathrm{OH}(92 \%)$ с соотношением компонентов $1: 0.5: 0.25$. Длительность травления составляла 20 мин. После первого и второго этапов пластина отмывалась в дистиллированной воде с целью удаления следов технологических растворов. На заключительном этапе образец отмывался в течение 15 мин в концентрированной азотной кислоте для удаления серебряных частиц из пористой пленки. Для проведения электрических измерений формировались In омические контакты к пленке por-Si и $\mathrm{Si}$-подложке на противоположных поверхностях полупроводниковой структуры.

С целью уточнения особенностей микроструктуры пленки por-Si применялся метод исследования спектра комбинационного рассеяния света (КРС). Измерялись спектры КРС пленки por-Si и монокристаллической подложки, используемой для изготовления экспериментального образца вблизи линии первого порядка КРС кремния $\left(520.6 \mathrm{~cm}^{-1}\right)$. Для измерения спектров КРС использовался спектрометр U-1000 (Jobin Ivon). Возбуждение КРС осуществлялось излучением Ar-лазера на длине волны 488 нм, направленным вдоль нормали к поверхности образца.

Особенности механизмов токопереноса в полупроводниковой структуре изучались с помощью измерения вольт-амперных характеристик (BAX) при различных температурах. Для измерений использовалась экспериментальная установка на базе гелиевого криостата замкнутого типа Janis CCS 400/204N и электрометра Keithley-6517B со встроенным регулируемым источником постоянного напряжения. Измерения ВАХ проводились при прямом и обратном смещении в диапазоне температур $T=70-370 \mathrm{~K}$ с шагом $10 \mathrm{~K}$. Прямое смещение соответствует приложению отрицательного потенциала к контакту на поверхности слоя por-Si, положительного потенциала - к омическому контакту к $p$-Si на противоположной поверхности исследуемой структуры.

Для исследования глубоких центров - ловушек носителей заряда с глубокими энергетическими уровнями (ГУ) применялся метод токовой релаксационной спектроскопии глубоких уровней (I-DLTS). Применялся I-DLTS-спектрометр, специально адаптированный для изучения образцов с относительно большой площадью (электрической барьерной емкостью), с высокими токами утечки [9], что характерно для изучаемой полупроводниковой структуры.

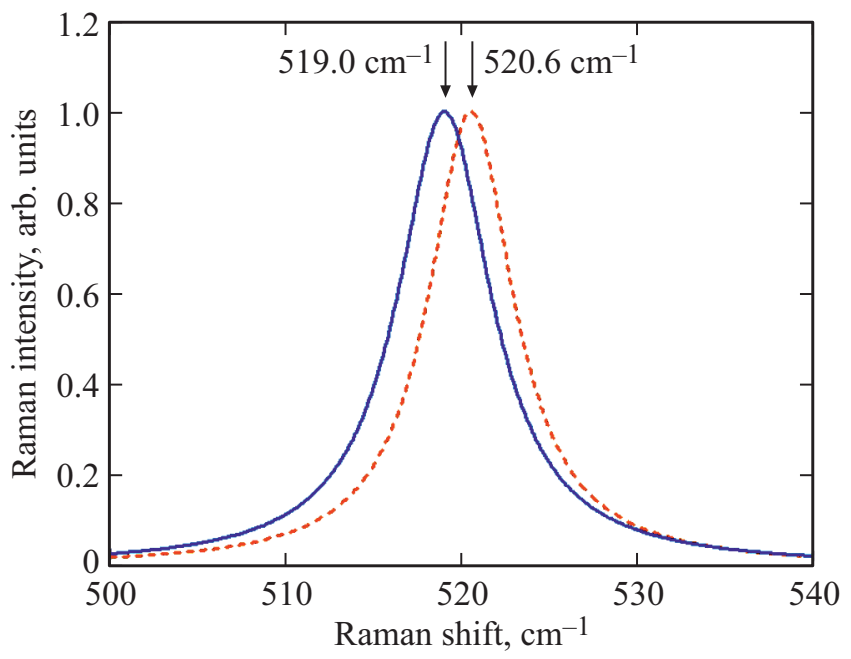

Рис. 1. Спектры комбинационного рассеяния света пленки por-Si (сплошная линия) и монокристаллической кремниевой подложки (пунктирная линия) экспериментального образца.

\section{3. Эксперимент и анализ результатов}

\section{1. Результаты исследования спектров комбинационного рассеяния света}

Спектры КРС поверхности пленки por-Si и кремниевой подложки показаны на рис. 1. Положение спектрального максимума линии первого порядка КРС подложки соответствует частоте $520.6 \mathrm{~cm}^{-1}$, что характерно для монокристаллического кремния. Для пленки por-Si по сравнению с подложкой линия первого порядка КРС смещена в область низких частот на $1.6 \mathrm{~cm}^{-1}$, при этом форма линии остается симметричной (рис. 1). Ширина рассматриваемой спектральной линии, определенная на уровне 0.5 от максимальной интенсивности, для пленки por-Si на $0.6 \mathrm{~cm}^{-1}$ больше, чем для подложки. Смещение спектральной линии КРС первого порядка в сторону нижних частот, сопровождающееся ее расширением в низкочастотную область, связывают с проявлением эффекта пространственного ограничения фононов в кремниевых кристаллитах пленки por-Si [10-12]. В нашем случае величины сдвига спектральной линии первого порядка КРС в низкочастотную область и уширения небольшие, следовательно, эффект пространственного ограничения фононов в пленке por-Si не проявляется.

Наиболее вероятной причиной, объясняющей наблюдаемое поведение спектров КРС (рис. 1), является возникновение растягивающих механических напряжений в результате формирования пленки por-Si исследуемой полупроводниковой структуры [13]. Рост пленки $p o r-\mathrm{Si}$ приводит к расширению кристаллической решетки кремния в направлении, перпендикулярном поверхности образца $[11,12]$. Деформация решетки в основном обусловлена разориентацией плоскостей, перпендикулярных поверхности пластины [12]. В работе [11] возникновение деформаций объясняется высоким отношением площади 
поверхности кремниевых кристаллитов пленки por-Si к их объему.

Величину механического напряжения $\sigma$ в пленке por-Si можно оценить с помощью формулы

$$
\sigma=-52.7 \Delta \omega
$$

где $\Delta \omega=\omega-\omega_{0}, \omega_{0}$ и $\omega-$ положения максимумов спектральной линии первого порядка КРС кремния при отсутствии и наличии механических напряжений соответственно, значение $\sigma$ выражено в МПа [13]. Для исследуемого образца $\omega=519.0 \mathrm{~cm}^{-1}, \omega_{0}=520.6 \mathrm{~cm}^{-1}$, значение $\sigma$ составило $84.3 \mathrm{MPa}$.

\section{2. Результаты исследования вольт-амперных характеристик}

Вольт-амперные характеристики исследуемой полупроводниковой структуры при различных температурах для прямого и обратного смещения представлены на рис. 2 и 3 соответственно в виде зависимостей плотности тока $J$ от приложенного постоянного напряжения смещения $U$. Для прямого смещения ветви BAX представлены в двойном логарифмическом масштабе (рис. 2), для обратного - в линейном масштабе (рис. 3). Из рис. 2 и 3 следует, что ВАХ исследуемой полупроводниковой структуры имеет выпрямляющий характер. Величина коэффициента выпрямления, определенная как отношение значений плотностей токов при $U=1.0$ В для прямого и обратного смещения, в диапазоне температур 70-370 K снижается от 915.7 до 14.8. При $T=300 \mathrm{~K}$ коэффициент выпрямления составляет 72.6 .

На прямых ветвях ВАX (рис. 2) можно выделить 4 участка, которые приблизительно аппроксимируются отрезками прямых, что свидетельствует о проявлении нескольких различных механизмов транспорта носителей заряда (токопереноса) при прямом смещении.

Участок 1 прямых ветвей BAX (рис. 2) может быть представлен экспоненциальной зависимостью, характерной для барьерной структуры:

$$
J \propto \exp \left(\frac{q U}{n k T}\right),
$$

где $q$ - элементарный заряд, $n-$ фактор неидеальности, $k$ - постоянная Больцмана [14]. Во всем исследованном диапазоне температур $n \approx 2$, следовательно, на участке 1 прямых ветвей $\mathrm{BAX}$ электрический ток определяется рекомбинацией носителей в области пространственного заряда.

Участок 2 прямых ветвей BAX может быть описан моделью тока, ограниченного пространственным зарядом (ТОПЗ), для которой характерна зависимость

$$
J \propto U^{m}
$$

где $m$ - показатель степени, характеризующий наклон соответствующего участка ВАХ [15]. В диапазоне температур $70-250 \mathrm{~K} m>2$, что с точки зрения модели

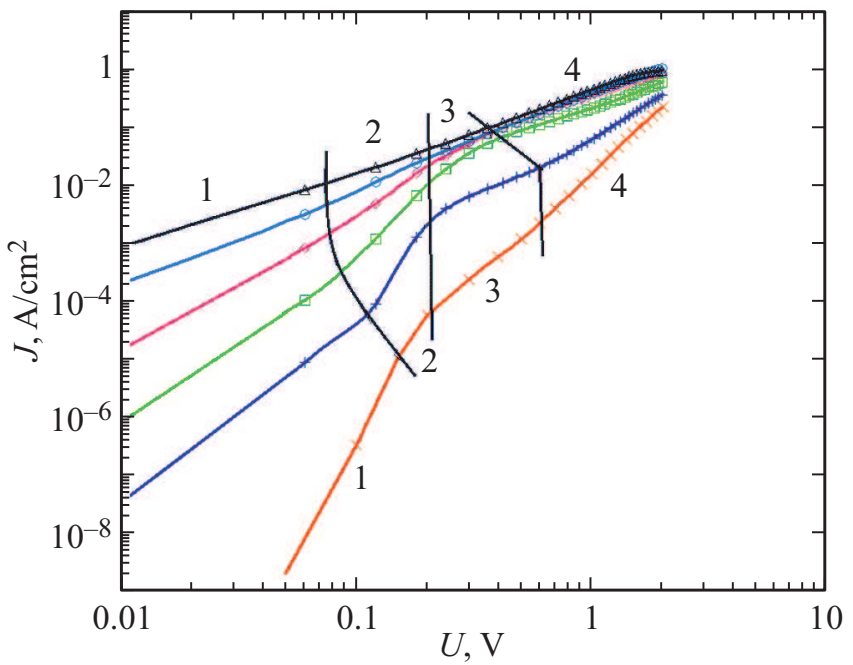

Рис. 2. Прямые ветви ВАХ при температуре $T, \mathrm{~K}: \times-70$, $+-140, \square-200, \diamond-250, \circ-300, \triangle-370$.

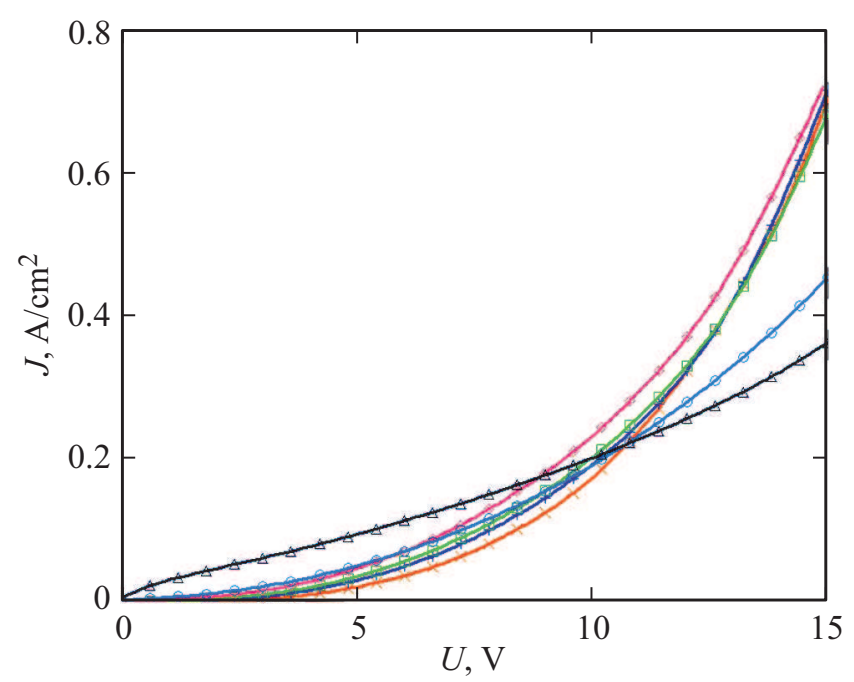

Рис. 3. Обратные ветви ВАХ при температуре $T, \mathrm{~K}: \times-70$, $+-140, \square-200, \diamond-250, \circ-300, \triangle-370$.

ТОП3 свидетельствует о влиянии на процессы токопереноса ловушек с экспоненциальным распределением по энергии активации [15]. При $T=260-290 \mathrm{~K} m=2$, что в рамках модели ТОП3, согласно [15], соответствует так называемому квадратичному ловушечному закону и характеризует влияние моноэнергетического глубокого центра (ловушки) на процессы токопереноса. В диапазоне температур 300-370 K на участке 2 прямых ветвей ВАХ (рис. 2) выполняется условие $1<m<2$. Согласно модели ТОПЗ это означает, что концентрация инжектированных носителей заряда становится сравнимой с концентрацией термически генерированных с ловушек носителей заряда [15].

На участках 3 и 4 прямых ветвей $\mathrm{BAX}$ (рис. 2) имеет место слабая зависимость величины $d(\ln J) / d U$ от температуры. Это свидетельствует о доминировании тун- 


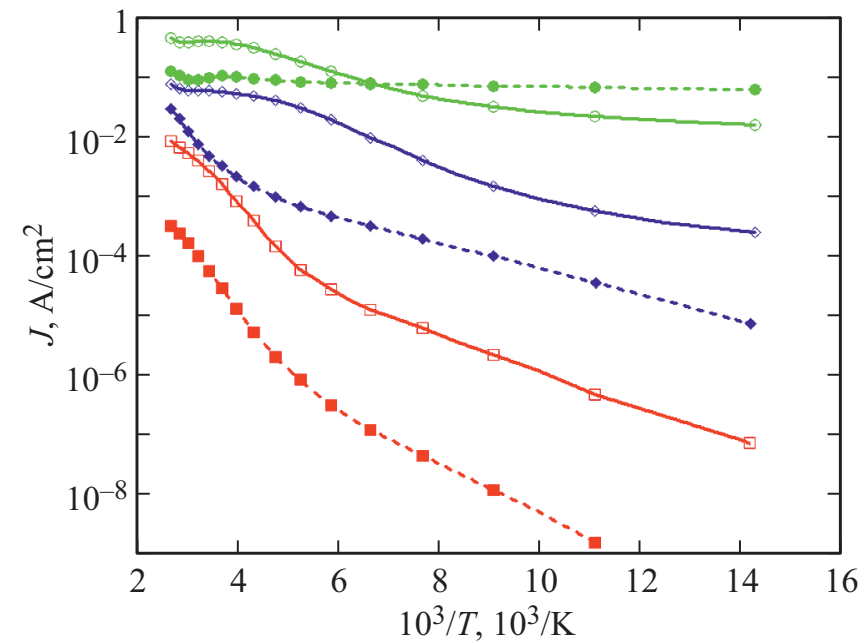

Рис. 4. Зависимость плотности тока от температуры при $U=0 \mathrm{~B}(\mathbf{\square})$, а также при прямом смещении для значений $U, \mathrm{~B}$ : $\square-0.1, \diamond-0.3, \circ-1.0$ и обратном смещении для значений $U, \mathrm{~B}:-1.0, \bullet-7.0$.

нельного механизма токопереноса [16]. Согласно [17], туннелирование носителей заряда может происходить внутри пленки por-Si между энергетическими уровнями ловушек на поверхности кремниевых кристаллитов через барьеры $\mathrm{SiO}_{x}$. Различие наклонов участков 3 и 4 BAX (рис. 2) может свидетельствовать о влиянии на процессы туннелирования групп ловушек с различающимися энергиями активации.

Обратные ветви ВАХ, представленные на рис. 3 в линейном масштабе, по качественному виду характерны для барьерных структур с так называемым „мягким“ пробоем [18]. В области обратного смещения слабая зависимость величины $J$ от температуры наблюдается при $U \leq 5 \mathrm{~B}$, что также свидетельствует о туннелировании носителей заряда. Таким образом, согласно [18], вид обратных ветвей ВАХ определяется конкуренцией лавинного и туннельного пробоя с участием ловушек $\mathrm{c}$ непрерывным диапазоном величин энергии активации.

На рис. 4 представлены графики температурной зависимости плотности тока при разных значениях $U$ для прямого и обратного смещения, а также при $U=0 \mathrm{~B}$. Температурная зависимость $J(U=0 \mathrm{~B})$ получена экстраполяцией прямых ветвей $\mathrm{BAX}$ к указанному значению $U$. При $U=0 \mathrm{~B}$ энергия активации ловушек изменяется в пределах $0.077-0.212$ эВ (рис. 4). В области прямого смещения при $U=0.1 \mathrm{~B}$ диапазон изменения энергии активации составляет $0.061-0.181$ эВ, при $U=0.3 \mathrm{~B}$ диапазон сдвигается в сторону меньших значений $0.008-0.073$ эВ. Наблюдаемое снижение энергии активации с ростом $U$ можно объяснить особенностью распределения ловушек, которая характерна для поверхностных состояний на кремниевых кристаллитах в пленке por-Si. При $U=1 \mathrm{~B}$ (прямое смещение) в области высоких и низких температур (рис. 4) $J$ слабо зависит от температуры, что может быть связано с отсутствием влияния перезарядки ловушек на процессы токопереноca, или с понижением высоты потенциального барьера, которое сопровождает эффект туннелирования носителей заряда. В области обратного смещения при $U=1$ В электрически активными являются состояния с энергией активации в диапазоне $0.045-0.254$ эВ, что также может объясняться влиянием поверхностных состояний (рис. 4). При $U=7 \mathrm{~B}$ в области обратного смещения (рис. 4) $J$ слабо зависит от температуры, что также может быть объяснено отсутствием влияния ловушек на процессы токопереноса или эффектом туннелирования носителей заряда.

\section{3. Результаты исследования экспериментальных образцов методом токовой релаксационной спектроскопии глубоких уровней}

При измерении I-DLTS-спектров заполнение ловушек проводилось импульсом напряжения с амплитудой $0 \mathrm{~B}$, опустошение ГУ осуществлялось при напряжении обратного смещения 2 В. При таком режиме происходит преимущественно заполнение ГУ ловушек основных носителей заряда - дырок. Выбор режима измерения обусловлен ограничением на максимально возможную величину обратного тока утечки через образец для используемого I-DLTS-спектрометра [9].

Спектры I-DLTS, измеренные при значениях времени релаксации $\tau$ 0.46, 0.91 и 1.41 мс, представлены на рис. 5. На спектре присутствует пик ГУ Н1 с энергией активации $E_{t}=0.46$ эВ, которая была определена по углу наклона прямой Аррениуса. Концентрация ловушек с ГУ Н1, определенная по высоте пика в соответствии с методикой, предложенной в [19], составила $1.37 \cdot 10^{16} \mathrm{~cm}^{-3}$. Ha I-DLTS-спектре со стороны низких температур присутствует уширенное „плечо“ в области температур 130-205 K, положение которого практически не зависело от постоянной времени $\tau$ (рис. 5). Это

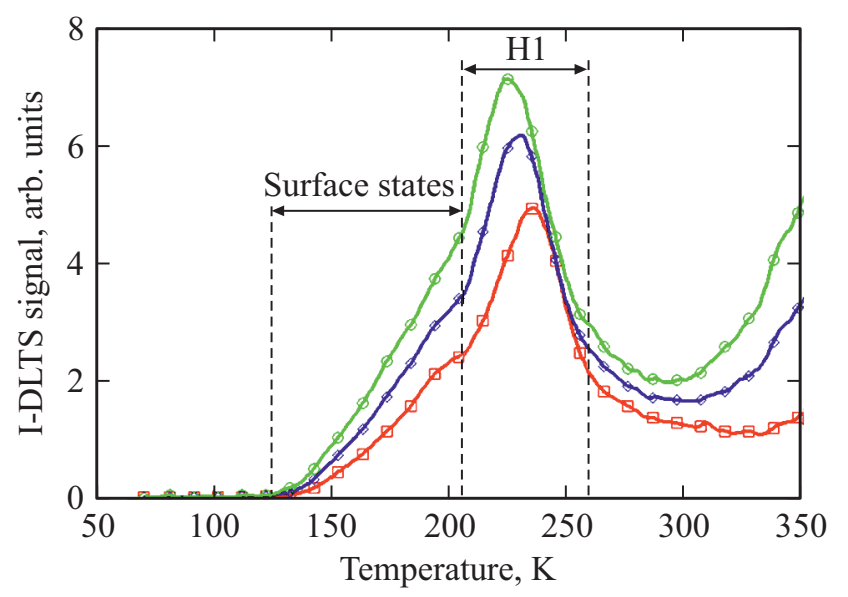

Рис. 5. Спектры I-DLTS экспериментального образца при постоянных времени $\tau$, мс: $\square-0.46, \diamond-0.91, \circ-1.41$. 
характерно для образца с высокой плотностью поверхностных состояний [20]. В соответствии с методикой, предложенной в [20], установлено, что наблюдаемые поверхностные состояния характеризуются диапазоном энергий активации $0.23-0.35$ эВ, величина плотности состояний составляет $4.96 \cdot 10^{11} \mathrm{~cm}^{-2}$. Наблюдаемые поверхностные состояния и ГУ Н1 в исследуемой полупроводниковой структуре являются ловушками основных носителей заряда - дырок. Важно отметить, что концентрация ГУ Н1 и плотность поверхностных состояний сравнимы с концентрацией мелкой акцепторной примеси $\left(1.5 \cdot 10^{16} \mathrm{~cm}^{-3}\right)$ в кремниевой пластине. Область при $T>300 \mathrm{~K}$, в которой наблюдается рост I-DLTS-сигнала, объясняется резким увеличением обратного тока утечки, на фоне которого становится невозможно выделить сигнал релаксации тока. Изменение интенсивности I-DLTSсигнала при изменении $\tau$ на рис. 5 связано с температурной зависимостью максимальной амплитуды тока релаксации в диодной структуре, в которой концентрация ловушек соизмерима с концентрацией основных носителей заряда.

\section{4. Обсуждение результатов эксперимента}

C помощью измерения знака термоэдс установлено, что пленка por-Si исследуемой полупроводниковой структуры имеет $p$-тип проводимости. Кремниевая подложка также имеет $p$-тип проводимости. Индиевые контакты к подложке и пленке por-Si являются невыпрямляющими (омическими). Исследования спектров КРС показали, что размерный эффект в кремниевых кристаллитах пленки por-Si не наблюдается. Следовательно, ширина запрещенной зоны $p$-Si подложки и кристаллитов пленки por-Si совпадает. Исходя из указанных соображений, ветви BAX при прямом и обратном смещении должны быть симметричными, в результате чего исследуемая полупроводниковая структура не должна проявлять выпрямляющих свойств. В то же время исследования показали, что в прямом смещении обнаруживается участок BAX, описываемый формулой (2), характерный для барьерных структур с эффектом выпрямления.

Для объяснения процессов токопереноса в исследуемой полупроводниковой структуре можно предложить модель зонной диаграммы для равновесного случая в виде рис. 6.

При формировании пленки por-Si возникает область с высокой концентрацией дефектов, сравнимой с концентрацией мелкой легирующей примеси в исходной пластине $p$ - $\mathrm{Si}$, используемой в качестве подложки. Как было установлено в результате измерения спектров I-DLTS, на границе раздела $p o r-\mathrm{Si} / p$-Si присутствуют поверхностные состояния, а также дискретный ГУ Н1. Косвенным образом возникновению дефектов с ГУ в кремниевых кристаллитах пленки por-Si может способствовать наличие механических напряжений [21] в por-Si, которые обнаружены при исследовании спектров КРС. Указанная

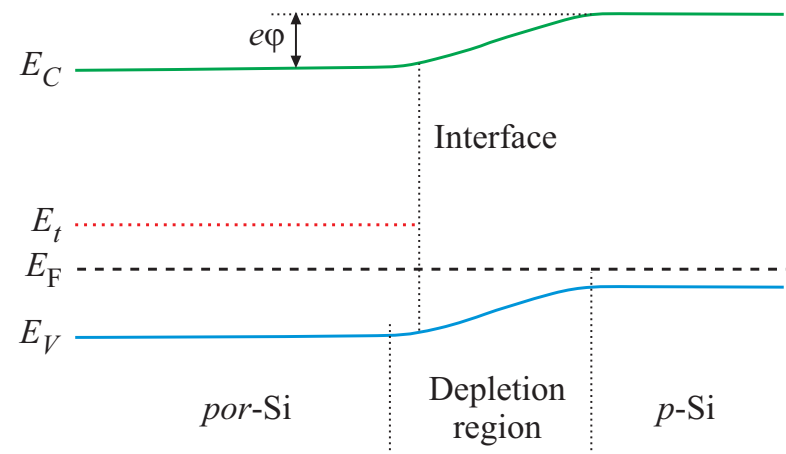

Рис. 6. Модель зонной диаграммы выпрямляющего перехода por- $\mathrm{Si} / p-\mathrm{Si}$.

область может представлять собой частично компенсированный полупроводник. В результате на границе между por-Si и $p$-Si уровень Ферми может быть зафиксирован со стороны por-Si. Область пространственного заряда (ОПЗ) исследуемой структуры находится в $p$-Si. Показанное на рис. 6 направление изгиба зон в ОПЗ обусловлено наличием эффекта выпрямления [14]. Величина изгиба зон на рис. 6 определяется высотой барьера еө. При приложении обратного напряжения смещения значение $е \varphi$ возрастает на величину $U$. При прямом смещении ец уменьшается. Указанными обстоятельствами можно объяснить выпрямляющие свойства исследуемой полупроводниковой структуры.

В результате исследования температурной зависимости ВАХ установлено, что процессы токопереноса носят сложный характер. При прямом смещении преобладают процессы рекомбинации носителей заряда в ОПЗ исследуемой полупроводниковой структуры, также проявляются механизм ТОПЗ и туннелирование носителей заряда внутри пленки por-Si между энергетическими уровнями ловушек на поверхности кремниевых кристаллитов через барьеры $\mathrm{SiO}_{x}$. При обратном смещении имеет место пробой барьерной структуры, определяющийся конкуренцией лавинного и туннельного механизмов с участием ловушек. Как при прямом, так и при обратном смещении на процессы токопереноса оказывают влияние ловушки с ГУ с энергией активации, распределенной в широком диапазоне значений.

\section{4. Заключение}

Показано, что структура с пленкой por-Si, выращенной методом металл-стимулированного травления с использованием частиц серебра на кремниевой монокристаллической пластине $p$-типа, обладает выпрямляющими свойствами. Так как эффект ограничения фононов в кристаллитах пористой пленки не наблюдался, то это означает отсутствие размерного эффекта в пленке por-Si, а также совпадение величин ширины запрещенной зоны por-Si и монокристаллического $p$-Si. Выпрямляющие свойства 
полупроводниковой структуры $p o r-\mathrm{Si} / p-\mathrm{Si}$ можно объяснить наличием изгиба энергетических зон вследствие фиксации уровня Ферми в слое por-Si из-за высокой концентрации глубоких центров. Исследование температурной зависимости ВАХ позволило выделить четыре участка на прямой ветви. При низких напряжениях (до $0.1 \mathrm{~B}$ ) наблюдается экспоненциальная зависимость тока, обусловленного процессами рекомбинации, характерная для полупроводниковых барьерных структур. С ростом напряжения ВАХ описывается моделью тока, ограниченного пространственным зарядом. При более высоких напряжениях наблюдается слабая зависимость величины от температуры, что свидетельствует о доминировании туннельного механизма токопереноса. Вид обратных ветвей ВАХ определяется вкладами лавинного и туннельного пробоев с участием ловушек. На спектрах релаксационной спектроскопии глубоких уровней наблюдаются пики, соответствующие глубокому уровню с энергией активации 0.46 эВ и электрически активным поверхностным состояниям с диапазоном энергий активации $0.23-0.35$ эВ. Наблюдаемые поверхностные состояния и дискретный ГУ в исследуемой полупроводниковой структуре являются ловушками основных носителей заряда - дырок.

\section{Финансирование работы}

Работа выполнена в рамках государственного задания Министерства науки и высшего образования РФ (FSSN2020-0003) с использованием оборудования Регионального центра зондовой микроскопии коллективного пользования при Рязанском государственном радиотехническом университете им. В.Ф. Уткина.

\section{Конфликт интересов}

Авторы заявляют, что у них нет конфликта интересов.

\section{Список литературы}

[1] T. Dzhafarov, A. Bayramov. In: Handbook of Porous Silicon, ed. by L. Canham (Springer International Publishing AG, part of Springer Nature, 2018) p. 1479.

[2] X. Liu, P.R. Coxon, M. Peters, B. Hoex, J.M. Cole, D.J. Fray. Energy Environ. Sci., 7, 3223 (2014).

[3] M. Karanam, G.M. Rao, S. Habibuddin, R. Padmasuvarna. Int. Lett. Chem., Phys. Astron., 71, 40 (2016).

[4] K.W. Kolasinski. In: Porous Silicon: From Formation to Application. Formation and Properties, ed. by G. Korotcenkov (London-N.Y., Taylor and Francis Group, LLC, 2016) v. 1 , p. 291.

[5] Ю.А. Жарова, В.А. Толмачев, С.И. Павлов. ФТП, 53 (4), 576 (2019).

[6] H. Han, Z. Huang, W. Lee. Nano Today, 9 (3), 271 (2014).

[7] H.V. Bandarenka. In: Handbook of Porous Silicon, ed. by L. Canham (Springer International Publishing AG, part of Springer Nature, 2018) p. 1315.
[8] Y. Zhao, Z. Liu, C. Liang, M. Yu. Maximov, B. Liu, J. Wang, F. Yin. Int. J. Electrochem. Sci., 12, 8591 (2017).

[9] А.В. Ермачихин, В.Г. Литвинов. ПТЭ, 2, 118 (2018).

[10] M. Yang, D. Huang, P. Hao. J. Appl. Phys., 75 (1), 651 (1994).

[11] M. Ivanda. In: Handbook of Porous Silicon, ed. by L. Canham (Springer International Publishing AG, part of Springer Nature, 2018) p. 611.

[12] G. Amato. In: Porous Silicon: From Formation to Application. Formation and Properties, ed. by G. Korotcenkov (LondonN.Y., Taylor and Francis Group, LLC, 2016) v. 1, p. 156.

[13] Qiu Li, Wei Qiu, Haoyun Tan, Jiangang Guo, Yilan Kang. Opt. Lasers Eng., 48 (11), 1119 (2010).

[14] С. Зи. Физика полупроводниковых приборов (М., Мир, 1984).

[15] М. Ламперт, П. Марк. Инжекционные токи в твердых meлаx (М., Мир, 1973).

[16] Б.Л. Шарма, Р.К. Пурохит. Полупроводниковые гетеропереходы (М., Сов. радио, 1979).

[17] А.А. Евтух, Э.Б. Каганович, Э.Г. Манойлов, Н.А. Семененко. ФТП, 40 (2), 180 (2006).

[18] В.В. Пасынков, Л.К. Чиркин. Полупроводниковые прибоpы (М., Высш. шк., 1987).

[19] V.G. Litvinov, N.V. Vishnyakov, V.V. Gudzev, N.B. Rybin, D.S. Kusakin, A.V. Ermachikhin, S.M. Karabanov, S.P. Vikhrov, A.S. Karabanov, E.V. Slivkin. MRS Advances, 1 (14), 911 (2016).

[20] Г.С. Дорджин, В.Н. Лактюшкин, М.В. Сорокина. Обзоры по электрон. техн., 7 (4), 72 (1989).

[21] К. Рейви. Дефекты и примеси в полупроводниковом кремнии (М., Мир, 1984).

Редактор Г.А. Оганесян

\section{Current Transfer in a Semiconductor Structure with a Porous Silicon Film formed by Metal-Stimulated Etching}

\author{
N.N. Melnik ${ }^{1}$, V.V. Tregulov ${ }^{2}$, V.G. Litvinov ${ }^{3}$, \\ A.V. Ermachikhin ${ }^{3}$, E.P. Trusov ${ }^{3}$, \\ G.N. Skoptsova ${ }^{2}$, A.I. Ivanov ${ }^{2}$ \\ ${ }^{1}$ Lebedev Physical Institute \\ Russian Academy of Sciences, \\ 119991 Moscow, Russia \\ ${ }^{2}$ Ryazan State University named for S.A. Yesenin, \\ 390000 Ryazan, Russia \\ ${ }^{3}$ Ryazan State Radio Engineering University \\ named after V.F. Utkin, \\ 390005 Ryazan, Russia
}

Abstract It is shown that during a porous Si film formation by metal-stimulated etching a barrier layer is formed on a monocrystal $p$-Si substrate. The rectifying properties of the semiconductor structure can be explained by the fixation of the Fermi level in the near-surface layer of porous $\mathrm{Si}$ due to a high concentration of electrically active defects (deep centers or traps). It causes to energy bands bending and the appearance of a potential barrier. The study of Raman scattering showed the absence of size effects and a change in the band gap in the porous $\mathrm{Si}$ film. Activation energies of deep centers by the temperature dependence of the current-voltage characteristics and deep level transient spectroscopy study were determined. 